\title{
Controlled permeation of model drugs through a bioconjugated membrane with antigen-antibody complexes as reversible crosslinks
}

\author{
Takashi Miyata $^{1,2,3}$, Noriko Asami ${ }^{1}$, Yurie Okita ${ }^{1}$ and Tadashi Uragami ${ }^{1,3}$ \\ Polymer Journal (2010) 42, 834-837; doi:10.1038/pj.2010.76; published online 1 September 2010
}

Keywords: antigen-antibody complex; bioconjugation; crosslink; membrane; network; stimuli-responsive hydrogel

\section{INTRODUCTION}

Biomembranes perceive specific ions and biomolecules, and then induce structural changes in them. To construct self-regulated drug delivery systems (DDS) by mimicking natural feedback systems, some researchers have developed stimuli-responsive membranes that change their permeability in response to $\mathrm{pH}$ and temperature changes. ${ }^{1-3}$ Only a few studies of stimuli-responsive membranes that respond to a target molecule have been undertaken, in spite of their potential applications in biochemical and biomedical fields. ${ }^{4-6}$ For example, a molecular recognition ion gating membrane was prepared by grafting a temperature-responsive polymer with crown ether moieties onto the surface of a porous polyethylene film by plasma graft copolymerization. ${ }^{5,6}$ The molecular recognition ion gating membrane spontaneously controlled the solution flux in response to a specific ion, such as $\mathrm{Ba}^{2+}$, which can be recognized by the crown ether moiety.

Some hydrogels exhibit a unique tendency to undergo abrupt changes in volume in response to environmental changes such as $\mathrm{pH}$ and temperature. ${ }^{7}$ Such hydrogels, called stimuli-responsive hydrogels, are smart materials that are promising candidates for designing novel DDS, switches and sensors. A few research teams have developed stimuli-responsive hydrogels that undergo a change in their volume in response to a target biomolecule (biomoleculeresponsive hydrogels)..$^{8-10}$ We proposed a strategy for designing biomolecule-responsive hydrogels that recognize target biomolecules such as saccharides and proteins. ${ }^{11-17}$ For example, the antigenresponsive hydrogels prepared using antigen-antibody complexes as reversible crosslinks swelled in the presence of a target antigen. ${ }^{14-16}$ Furthermore, glycoprotein-responsive hydrogels, which shrunk in response to the target tumor marker glycoprotein, were also prepared by molecular imprinting, using lectins and antibodies as ligands. ${ }^{17}$ Such biomolecule-responsive hydrogels are highly promising materials for constructing self-regulated DDS and diagnostic systems.
To achieve controlled permeation of a drug in response to a target antigen, as shown in Figure 1, we investigated the permeation behavior of two model drugs with different molecular weights, vitamin $\mathrm{B}_{12}$ $\left(M=1355 \mathrm{~g} \mathrm{~mol}^{-1}\right)$ and hemoglobin $\left(M=64500 \mathrm{~g} \mathrm{~mol}^{-1}\right)$, using a bioconjugated membrane with antigen-antibody complexes as reversible crosslinks (that is, the antigen-antibody membrane), which exhibited reversible, antigen-responsive swelling/shrinking behavior. This paper discusses the effect of antigen concentration in a buffer solution on the permeability of a drug through a membrane, which is based on the relationship between network size and drug size. Investigations of permeations of drugs with different molecular weights will contribute significantly to our understanding of a change in the network size of the antigen-antibody membrane in response to a target antigen.

\section{EXPERIMENTAL PROCEDURE}

\section{Materials}

Materials included a rabbit immunoglobulin G (rabbit IgG), which was used as an antigen, and a goat anti-rabbit immunoglobulin G (goat anti-rabbit IgG), which was used as an antibody; these were purchased from Funakoshi (Tokyo, Japan). The goat anti-rabbit IgG was purified with a ProtOnTM Kit 1 (Multiple Peptide Systems, San Diego, CA, USA). In the purification method, the rabbit IgG was immobilized on the ProtOnTM column, which contained agarose resin that had been modified with an $N$-hydroxyl succinimide ester. Thereafter, the goat anti-rabbit IgG was purified through the resultant affinity ProtOnTM column.

Acrylamide (AAm; Wako Pure Chemical Industries, Osaka, Japan) was purified by recrystallization from toluene $/ n$-hexane. $N$-succinimidyl acrylate (NSA) was purchased from Acros Organics (Morris Plains, NJ, USA) and used as received. $N, N, N^{\prime}, N^{\prime}$-Tetramethylethylenediamine, ammonium persulfate and $N, N^{\prime}$-methylenebisacrylamide were purchased from Wako Pure Chemical Industries and used as received. All other solvents and reagents were of analytical grade, and were obtained from commercial sources without further purification.

\footnotetext{
${ }^{1}$ Department of Chemistry and Materials Engineering, Kansai University, Suita, Osaka, Japan; 2PRESTO, Japan Science and Technology Agency, Kawaguchi, Saitama, Japan and ${ }^{3}$ High Technology Research Center, Kansai University, Suita, Osaka, Japan 


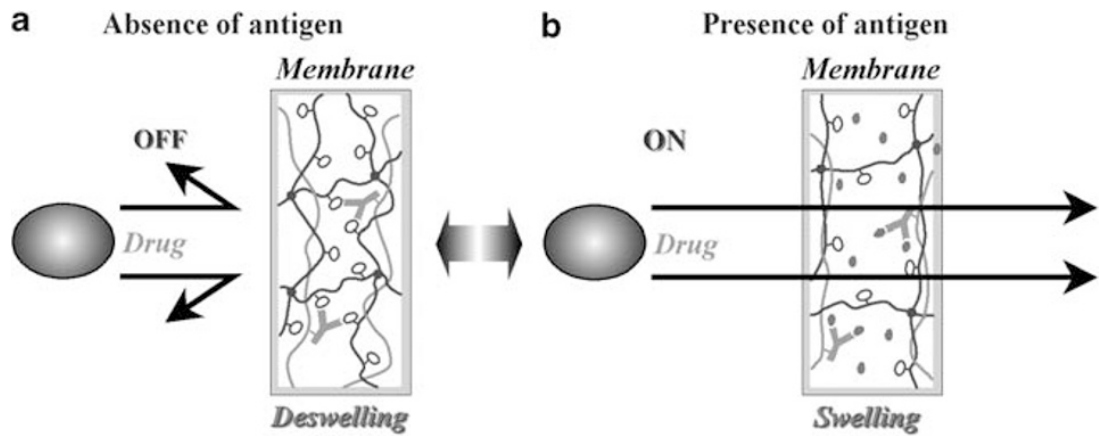

Figure 1 Schematic of controlled permeation of a drug through an antigen-antibody membrane in response to a target antigen.

1) Introduction of an acryloyl group into antigne and antibody

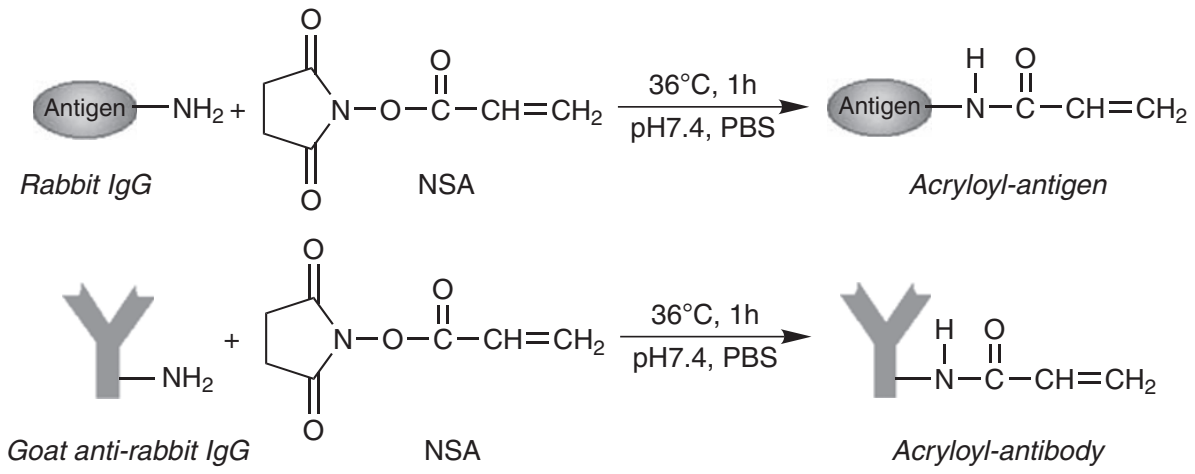

2) Coplymerization of acryloyl-antigen and acrylamide

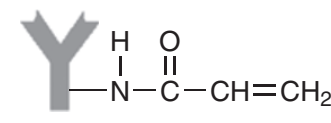

Acryloyl-antibody

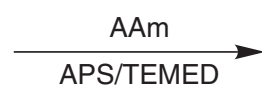

3) Preparation of an antigen-antibody membrane

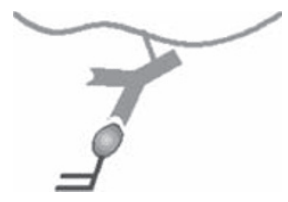

Antigen-antibody complex

\section{$\underset{\mathrm{APS} / \mathrm{TEMED}}{\stackrel{\text { AAm, MBAA }}{\longrightarrow}}$}

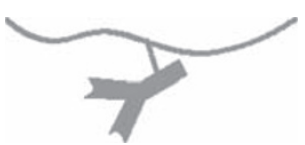

PAAm-grafted antibody

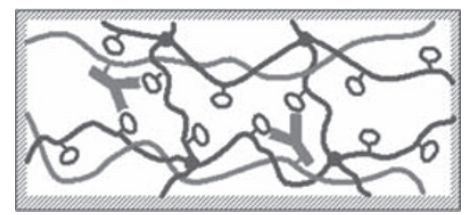

Antigen-antibody membrane

Scheme 1 Preparation of a bioconjugated membrane having antigen-antibody complexes as reversible crosslinks in a semi-interpenetrating polymer network. AAm, acrylamide; APS, ammonium persulfate; IgG, immunoglobulin G; MBAA, $N, N^{\prime}$-methylenebisacrylamide; NSA, succinimidyl acrylate; PAAm, poly(acrylamide); PBS, phosphate buffer solution; TEMED, $N, N, N^{\prime}, N^{\prime}$-tetramethylethylenediamine.

\section{Modification of antigen and antibody}

An antigen was chemically modified for the purpose of introducing acryloyl groups in the following manner (Scheme 1): The rabbit IgG, as an antigen, was chemically modified by coupling it with NSA in a phosphate buffer solution using the method reported previously. ${ }^{16} \mathrm{NSA}(4 \mathrm{mg})$ was added to a phosphate buffer solution $(0.02 \mathrm{M}, \mathrm{pH} 7.4)$ containing the rabbit $\operatorname{IgG}(100 \mathrm{mg})$. The reaction was carried out at $36^{\circ} \mathrm{C}$ for $1 \mathrm{~h}$ to introduce acryloyl groups into the rabbit IgG. The resulting modified antigen (acryloyl-rabbit IgG) was purified by gel filtration to remove unreacted NSA. Chemically modified goat antirabbit IgG, as an antibody, was also synthesized by coupling it with NSA in a phosphate buffer solution, using the same modification method that was used on the antigen, as follows: NSA $(0.4 \mathrm{mg})$ was added to a phosphate buffer solution (0.02 M, pH 7.4) containing goat anti-rabbit IgG (100 mg) (NSA/ antibody ratio $=6(\mathrm{~mol} / \mathrm{mol}))$. Thereafter, the reaction mixture was incubated at $36^{\circ} \mathrm{C}$ for $1 \mathrm{~h}$ to introduce acryloyl groups into the goat anti-rabbit IgG. The resultant acryloyl-goat anti-rabbit IgG was purified by gel filtration, and a phosphate buffer solution containing acryloyl-goat anti-rabbit IgG was obtained at a concentration of $1.9 \mathrm{mg} \mathrm{ml}^{-1}$.

\section{Preparation of an antigen-antibody membrane}

An antigen-antibody membrane with a semi-interpenetrating polymer network (semi-IPN) structure, consisting of linear poly(acrylamide) (PAAm) chains with pendant antibodies and PAAm networks with pendant antigens, was 
strategically prepared as follows (Scheme 1$)^{16}$ : AAm $(30 \mathrm{mg}$ ) was added to $570 \mathrm{mg}$ of acryloyl-goat anti-rabbit IgG solution, along with $0.01 \mathrm{ml}$ of $0.1 \mathrm{M}$ aqueous ammonium persulfate solution and $0.01 \mathrm{ml}$ of $0.8 \mathrm{M}$ aqueous $N, N, N^{\prime}$, $N^{\prime}$-tetramethylethylenediamine solution, which functioned as redox initiators. Copolymerization was performed at $25^{\circ} \mathrm{C}$ for $3 \mathrm{~h}$ to synthesize PAAm-grafted goat anti-rabbit IgG. In addition, acryloyl-rabbit IgG $(2.46 \mathrm{mg}), \mathrm{AAm}(82 \mathrm{mg})$ as main monomer and $N, N^{\prime}$-methylenebisacrylamide $(0.1 \mathrm{wt} \%$ relative to $\mathrm{AAm})$ as a crosslinker were dissolved in $600 \mathrm{mg}$ of phosphate buffer solution containing PAAm-grafted goat anti-rabbit IgG to form an antigen-antibody complex. After adding $0.01 \mathrm{ml}$ of $0.1 \mathrm{M}$ aqueous ammonium persulfate and $0.01 \mathrm{ml}$ of $0.8 \mathrm{M}$ aqueous $N, N, N^{\prime}, N^{\prime}$-tetramethylethylenediamine to the mixture, it was injected into a mold with two glass plates that were separated by a $1 \mathrm{~mm}$ spacer. Polymerization was carried out at $25^{\circ} \mathrm{C}$ for $3 \mathrm{~h}$. The resulting antigen-antibody membrane was immersed in a phosphate buffer solution to remove residual chemicals and unreacted monomers. The PAAm membrane was also prepared as a reference through redox copolymerization of AAm and $N, N^{\prime}$-methylenebisacrylamide in the presence of linear PAAm in the same manner as the antigen-antibody membrane.

\section{Permeation experiments}

Permeations of vitamin $\mathrm{B}_{12}$ and hemoglobin through the antigen-antibody membrane, both in the absence and presence of a target antigen, were carried out at $25^{\circ} \mathrm{C}$ under magnetic stirring, using a diaphragm glass cell that consisted of two detachable parts. The membrane was placed between the two detachable parts of the cell, which were then clamped and sealed tightly with silicon rubber. The left-side chamber of the cell was filled with a phosphate buffer solution containing vitamin $\mathrm{B}_{12}\left(4 \mathrm{mg} \mathrm{ml}^{-1}\right)$ or hemoglobin $\left(1 \mathrm{mg} \mathrm{ml}^{-1}\right)$. The antigen concentration in the phosphate buffer solution placed in the chamber on the right was changed from 0 to $4 \mathrm{mg} \mathrm{ml}^{-1}$. The amounts of vitamin $B_{12}$ and hemoglobin that permeated through the membrane were determined colorimetrically from the absorbance at 360 and $405 \mathrm{~nm}$, respectively, using a spectrophotometer (Shimadzu, Kyoto, Japan; UV-2500PC).

\section{RESULTS AND DISCUSSION}

In previous papers, we discussed the effect of the preparation on the condition of the protein contents in the resultant antigen-antibody hydrogels, as well as on their swelling ratio. ${ }^{14-16}$ These papers revealed that antigen-antibody hydrogels undergo a change in their volume in response to a target antigen, but they do not respond to other antigens. The antigen-responsive swelling/shrinking behavior of the antigen-antibody hydrogels is attributed to the fact that formation and dissociation of the complex between antigen and antibody, which are linked with polymer networks, induce a change in the network crosslinking density.

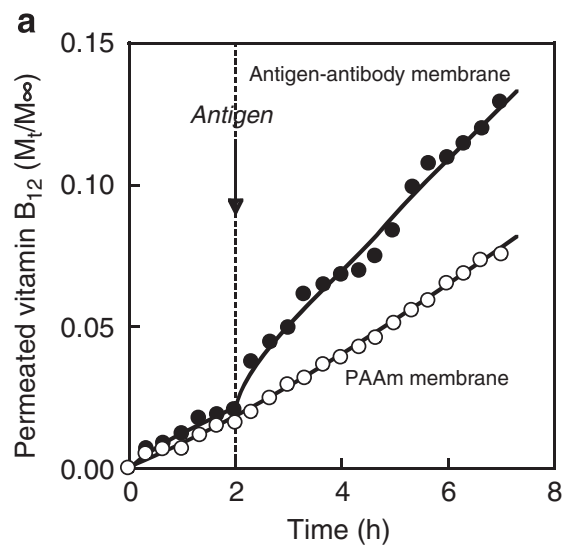

Figure 2 shows permeation profiles of vitamin $B_{12}$ and hemoglobin through PAAm and antigen-antibody membranes when a target antigen was added to a buffer solution after permeation in the absence of antigen for $2 \mathrm{~h}$. The relative amount of permeated vitamin $\mathrm{B}_{12}$ and hemoglobin at a certain time $t\left(M_{\mathrm{t}}\right)$ is normalized by the amount at infinite time $\left(M_{\infty}\right)$ and represented on the vertical axis. Before adding a target antigen, vitamin $B_{12}$ was permeated through PAAm and antigen-antibody membranes, but hemoglobin was not. Vitamin $B_{12}$ can diffuse more easily than hemoglobin in these membrane networks because the molecular size of vitamin $B_{12}$ is smaller than that of hemoglobin. Permeation profiles before antigen addition into a buffer solution demonstrate that the permeation rate of model drugs through the antigen-antibody membrane was the same as through the PAAm membrane. This implies that the antigen-antibody membrane had almost the same network size as the PAAm membrane, and that the cutoff molecular weight of their membranes was between 1355 and $64500 \mathrm{~g} \mathrm{~mol}^{-1}$. Furthermore, the permeability of vitamin $\mathrm{B}_{12}$ and hemoglobin through the antigen-antibody membrane was effectively enhanced by the addition of a target antigen into a buffer solution, but the permeability through the PAAm membrane did not change at all. The sharply regulated permeation of hemoglobin through the antigen-antibody membrane in response to a target antigen demonstrates that its network size, in the absence of an antigen, was smaller than the molecular size of hemoglobin, but that the network size in the presence of an antigen became larger than that of hemoglobin. It should be noted that the permeability of vitamin $\mathrm{B}_{12}$ through the antigen-antibody membrane was also enhanced by the presence of an antigen in spite of the small size of vitamin $B_{12}$. Previous papers reported that bioconjugated hydrogels, having antigen-antibody complexes as reversible crosslinks, undergo reversible changes in their volume in response to a target antigen. ${ }^{14-16}$ Therefore, we can conclude that the antigen-responsive change in the network size of the antigen-antibody membrane induced a change in the diffusion coefficient of vitamin $\mathrm{B}_{12}$ and hemoglobin in the membrane.

The relationship between the antigen concentration of a buffer solution and the permeation rate of hemoglobin through the antigenantibody membrane was examined after the addition of a target antigen into the buffer solution (Figure 3 ). The permeation rate in Figure 3 is defined as the relative amount of drug permeated per hour. The antigen-antibody membrane inhibited hemoglobin permeation effectively in the absence of an antigen. The permeation rate of

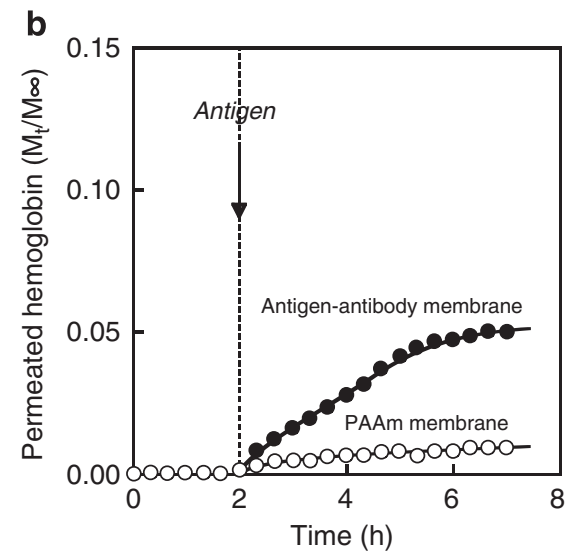

Figure 2 Permeation profile of vitamin $\mathrm{B}_{12}$ (a) and hemoglobin (b) through the poly(acrylamide) (PAAm) membrane ( $\left.\bigcirc\right)$ and antigen-antibody membrane ( $\bullet$ ) in the absence and presence of a target antigen. The antigen with a concentration of $4 \mathrm{mg} \mathrm{ml}^{-1}$ was added after $2 \mathrm{~h}$. 


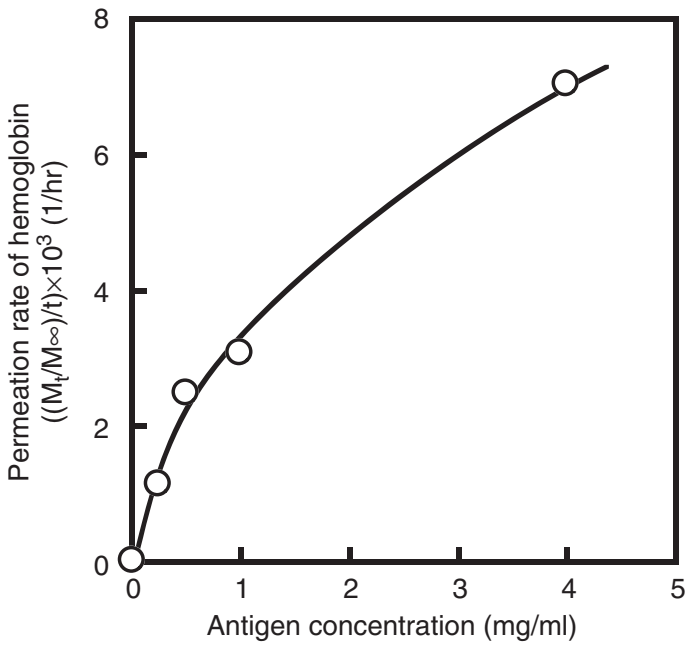

Figure 3 Effect of antigen concentration on the permeation rate of hemoglobin through the antigen-antibody membrane in a phosphate buffer solution containing a target antigen.

hemoglobin through the antigen-antibody membrane increased gradually with an increase in the antigen concentration of the buffer solution. Previous studies revealed that the swelling ratio of the antigen-antibody hydrogel is strongly dependent on the antigen concentration of the buffer solution. ${ }^{14}$ Therefore, hemoglobin permeability, which is enhanced in the presence of a target antigen, is attributed to an increase in the free volume induced by antigenresponsive expansion of the polymer network. In addition, the antigen-antibody complexes linked with the polymer network function as an obstacle for preventing hemoglobin from diffusing in the network. However, because the presence of a target antigen results in a dissociation of the antigen-antibody complexes that are linked with the network by a complex exchange mechanism, the diffusivity of hemoglobin in the membrane can be enhanced by a decrease in the crosslinks that consist of antigen-antibody complexes (Figure 1). A close correlation between antigen concentration and drug permeability not only provides a basis for the development of self-regulated DDS using an antigen-responsive membrane but also permits more insight into the structural changes of smart networks.

\section{CONCLUSIONS}

Permeation of model drugs with different molecular weights through a bioconjugated membrane that has antigen-antibody complexes as reversible crosslinks was investigated in both the absence and presence of a target antigen. Drug permeation through the bioconjugated membrane was suppressed in the absence of a target antigen but, in its presence, permeation became enhanced. This indicates that the bioconjugated membrane can regulate drug permeation in response to the concentration of a target antigen. The antigen-responsive permeation profiles of the drugs through the membrane were strongly influenced by the molecular weight of the drugs. The antigenresponsive change in the network size of the antigen-antibody membrane induced a change in the diffusion coefficient of the drugs through the membrane; this was because the presence of the antigen resulted in the dissociation of the antigen-antibody complexes that were functioning as reversible crosslinks. In addition, permeation experiments with drugs of different molecular weights demonstrated that the network size of the antigen-antibody membrane, when in the absence of a target antigen, was smaller than the molecular size of hemoglobin. However, the network size of the antigen-antibody membrane that was in the presence of a target antigen became larger than that of hemoglobin. Even though antigen-responsive membranes still require further research to find possible applications, they are likely to become an important material for constructing self-regulated DDS in the future.

\section{ACKNOWLEDGEMENTS}

This study was supported in part by Grants-in-aid for Scientific Research from the Ministry of Education, Culture, Sports, Science and Technology of Japan.

1 Park, Y. S., Ito, Y. \& Imanishi, Y. pH-Controlled gating of a porous glass filter by surface grafting of polyelectrolyte brushes. Chem. Mater. 9, 2755-2758 (1997).

2 Park, Y. S., Ito, Y. \& Imanishi, Y. Permeation control through porous membranes immobilized with thermosensitive polymer. Langmuir 14, 910-914 (1998).

3 Chu, L. Y., Yamaguchi, T. \& Nakao, S. A molecular-recognition microcapsule for environmental stimuli-responsive controlled release. Adv. Mater. 14, 386-389 (2002).

4 Ishihara, K., Kobayashi, M., Ishimaru, N. \& Shinohara, I. Glucose-induced permeation control of insulin through a complex membrane consisting of immobilized glucoseoxidase and a poly(amine). Polym. J. 16, 625-631 (1984).

5 Yamaguchi, T., Ito, T., Sato, T., Shinbo, T. \& Nakao, S. Development of a fast response molecular recognition ion gating membrane. J. Am. Chem. Soc. 121, 4078-4079 (1999).

6 Ito, T. \& Yamaguchi, T. Controlled release of model drugs through a molecular recognition ion gating membrane in response to a specific ion signal. Langmuir 22, 3945-3949 (2006)

7 Miyata, T. Stimuli-Responsive Polymers and Gels. in Supramolecular Design for Biological Applications (ed. Yui, N.) Ch. 9, 191-225 (CRC Press, Boca Raton, 2002).

8 Miyata, T., Uragami, T. \& Nakamae, K. Biomolecule-sensitive hydrogels. Adv. Drug Delivery Rev. 54, 79-98 (2002)

9 Miyata, T \& Uragami, T Biological stimuli-responsive hydrogels. in Polymeric Biomaterials (ed. Dumitriu, S.) Ch. 36, 959-974 (Marcel Dekker Inc, New York, 2002).

10 Miyata, T Biomolecule-responsive gels: design of smart gels using biomolecular interactions. in Bottom-Up Nanofabrication: Supramolecules, Self-Assemblies, and Organized Films (eds Ariga, K., Nalwa, H. S.) Vol. 6, Ch. 9, 137-150 (American Scientific Publishers, Los Angeles, 2009).

11 Nakamae, K., Miyata, T., Jikihara, A. \& Hoffman, A. S. Formation of poly(glucosyloxyethyl methacrylate)-concanavalin A complex and its glucose-sensitivity. J. Biomaterials Sci. Polym. Ed. 6, 79-90 (1994).

12 Miyata, T., Jikihara, A., Nakamae, K. \& Hoffman, A. S. Preparation of poly(glucosyloxyethyl methacrylate)-concanavalin A complex hydrogel and its glucose-sensitivity. Macromol. Chem. Phys. 197, 1135-1146 (1996).

13 Miyata, T., Jikihara, A., Nakamae, K. \& Hoffman, A. S. Preparation of reversibly glucose-responsive hydrogels by covalent immobilization of lectin in polymer networks having pendant glucose. J. Biomaterials Sci. Polym. Ed. 15, 1085-1098 (2004).

14 Miyata, T., Asami, N. \& Uragami, T. A reversibly antigen-responsive hydrogel. Nature 399, 766-769 (1999).

15 Miyata, T., Asami, N. \& Uragami, T. Preparation of an antigen-sensitive hydrogel using antigen-antibody bindings. Macromolecules 32, 2082-2084 (1999).

16 Miyata, T., Asami, N. \& Uragami, T. Structural design of stimuli-responsive bioconjugated hydrogels that respond to a target antigen. J. Polym. Sci. Polym. Phys. 47, 2144-2157 (2009).

17 Miyata, T., Jige, M., Nakaminami, T. \& Uragami, T. Tumor marker-responsive behavior of gels prepared by biomolecular imprinting. Proc. Natl. Acad. Sci. USA 103, 1190-1193 (2006). 